\title{
CONVOLUTIONAL RECURRENT NEURAL NETWORK AND DATA AUGMENTATION FOR AUDIO TAGGING WITH NOISY LABELS AND MINIMAL SUPERVISION
}

\author{
Janek Ebbers, Reinhold Haeb-Umbach \\ Paderborn University, Department of Communications Engineering, Paderborn, Germany \\ \{ebbers, haeb\}@nt.upb.de
}

\begin{abstract}
In this paper we present our audio tagging system for the DCASE 2019 Challenge Task 2. We propose a model consisting of a convolutional front end using log-mel-energies as input features, a recurrent neural network sequence encoder and a fully connected classifier network outputting an activity probability for each of the 80 considered event classes. Due to the recurrent neural network, which encodes a whole sequence into a single vector, our model is able to process sequences of varying lengths. The model is trained with only little manually labeled training data and a larger amount of automatically labeled web data, which hence suffers from label noise. To efficiently train the model with the provided data we use various data augmentation to prevent overfitting and improve generalization. Our best submitted system achieves a label-weighted label-ranking average precision (lwlrap) of $75.5 \%$ on the private test set which is an absolute improvement of $21.7 \%$ over the baseline. This system scored the second place in the teams ranking of the DCASE 2019 Challenge Task 2 and the fifth place in the Kaggle competition "Freesound Audio Tagging 2019" with more than 400 participants. After the challenge ended we further improved performance to $76.5 \%$ lwlrap setting a new state-of-the-art on this dataset.
\end{abstract}

Index Terms - audio tagging, label noise, data augmentation

\section{INTRODUCTION}

Environmental sound recognition has recently attracted increased interest, not only in academia. Numerous commercial applications can benefit from a reliable acoustic scene analysis, such as ambient assisted living, autonomous driving and various monitoring and diarization tasks. Within environmental sound recognition the tasks of Sound Event Detection (SED), Audio Tagging and Acoustic Scene Classification (ASC) [1] can be distinguished, which differ in the level of detail obtained about the acoustic environment. While SED makes predictions at frame level, Audio Tagging and ASC make predictions at sequence level.

Because frame level annotations (so-called strong labels) are difficult and time-consuming to obtain, current large-scale datasets, such as Google's AudioSet [2], only provide sequence level labels (weak labels). Further, for many applications frame level predictions are not required, which, together with the availability of weakly labeled large-scale datasets, results in an increased popularity of Audio Tagging.

Although weak annotations are easier to obtain than strong annotations, they still require human annotators. To avoid this necessity completely, one line of research is devoted to develop

This work has been supported by Deutsche Forschungsgemeinschaft under contract no. HA 3455/15-1 within the Research Unit FOR 2457 (acoustic sensor networks). semi-supervised approaches which directly benefit from unlabeled data $[3,4]$, which is abundant. However, there are also tremendous amounts of data coming with other modalities and meta-data, which can potentially be exploited to derive labels automatically. In the dataset considered in this contribution, labels had been generated for the web data using video-level predictions. Note, that those automatically generated labels certainly contain errors, which is why they are called noisy labels. Until now there are only few works addressing the impact of noisy labels for sound recognition [5].

Data augmentation is another common approach to increase the amount of labeled training data and improve generalization. It has been shown to improve classifier performance on many tasks, including speech recognition [6] and audio classification [7, 8, 9].

In this contribution we tackle the DCASE 2019 Challenge Task 2 [10], where the main research objective is the following: How can we train a high performance Audio Tagging system given relatively little data with reliable labels but a larger amount of mismatched data with noisy labels? We primarily tackle this by exploring different methods for data augmentation, while we spend less time on neural network architecture tuning. We show that frequency warping and time and frequency masking for data augmentation significantly improve performance. Further, our experiments tend to suggest, that our model is robust against label noise, as it achieves state-of-the-art performance without any particular treatment of label noise. While the methods investigated for dealing with label noise did individually not result in classifier improvements, they nevertheless increased the diversity of the models trained, resulting eventually in performance improvement through system combination. Please note that our system is publicly available on github. ${ }^{1}$

The rest of the paper is structured as follows. After briefly describing the considered task in Section 2, we outline our neural network architecture in Section 3. After presenting the data augmentation methods in Section 4, our training procedure and experiments are given in Sections 5 and 6, respectively. Conclusions are drawn in Section 7.

\section{TASK DESCRIPTION}

The DCASE 2019 Challenge Task 2 "Audio Tagging with noisy labels and minimal supervision" [10] is a follow-up of the DCASE 2018 Challenge Task 2 "General-purpose audio tagging of Freesound content with AudioSet labels" [11]. As in the 2018 edition the challenge was hosted on $\mathrm{Kaggle}^{2}$ with more than 400 participants. The provided dataset "FSDKaggle2019" consists of two subsets: a small curated set of 4970 manually labeled audio clips and a noisy set of 19815 audio clips where labels were automatically derived from video-level predictions from a variety of

\footnotetext{
${ }_{1}$ https://github.com/fgnt/upb_audio_tagging_2019

${ }^{2}$ https://www.kaggle.com/c/freesound-audio-tagging-2019
} 
pre-trained audio models. A vocabulary of 80 sound events is used where multiple events can be active at a time resulting in a multilabel classification problem.

\section{MODEL}

\subsection{Feature Extraction}

First, we perform an STFT with a frame length of $40 \mathrm{~ms}$ (1764 samples) and a hop size of $20 \mathrm{~ms}$ (882 samples) on the provided $44.1 \mathrm{kHz}$ audio signals without resampling. For each frame we then extract 128 log-mel-band-energy features with $f_{\min }=50 \mathrm{~Hz}$ and $f_{\max }=16 \mathrm{kHz}$. Lastly, we substract the global mean of each feature and then divide by the global standard deviation over all features.

\subsection{Neural Network Architecture}

Our proposed deep learning based model is outlined in Table 1. The neural network consists of a convolutional (conv.), a recurrent, and a fully connected module. We expect a four dimensional input to our model of shape $B \times C \times F \times N_{m}$ with $B, C, F, N_{m}$ being the mini-batch size, number of channels, number of features and number of frames in the $m$-th input signal, respectively, where $C=1$ and $F=128$ are fix. In the following the signal index $m$ is neglected.

The convolutional module combines a $2 \mathrm{~d} \mathrm{CNN}$ and a $1 \mathrm{~d} \mathrm{CNN}$. The $2 \mathrm{~d}$ CNN consists of five conv. blocks, with each block comprising one or two conv. layers and a max pooling layer. While the first four blocks have two conv. layers the last block only has a single one. In each block the number of channels is doubled starting from 16 while the number of features are halfed by max pooling. The number of time steps are also halfed in the first three blocks while being unchanged in the last two blocks. This results in an output of shape $B \times C^{\prime} \times F^{\prime} \times N^{\prime}$ with $C^{\prime}=256, F^{\prime}=4$ and $N^{\prime}=\left\lceil\frac{N}{8}\right\rceil$. Each $2 \mathrm{~d}$ conv. layer uses a kernel size of $3 \times 3$ and is followed by batch normalization and ReLU activation.

While the $2 \mathrm{~d} \mathrm{CNN}$ is meant to extract high-level feature maps from the log-mel-band-energy spectrogram, the $1 \mathrm{~d} \mathrm{CNN}$ (or TDNN) is meant to provide holistic representations by jointly processing all frequencies and channels of adjacent frames. Therefore it takes the reshaped output $\left(B \times C^{\prime} \cdot F^{\prime} \times N^{\prime}\right)$ of the $2 \mathrm{~d} C N N$ as input and applies three $1 \mathrm{~d}$ conv. layers with 256 hidden channels each. Each 1d conv. layer uses a kernel size of 3 and is followed by batch normalization and ReLU activation.

The output of the $\mathrm{CNN}$ is then fed into a recurrent sequence encoder. We use two layers of Gated Recurrent Units (GRUs) with 256 units per layer. Only the last output vector of each sequence in a batch is forwarded to the classification network.

The fully connected classification network conists of one hidden layer with 256 hidden units and ReLU activation function and the final classification layer with Sigmoid activation outputting scores between 0 and 1 for each of the 80 target event classes.

\section{DATA AUGMENTATION}

Because there is only little data available, efficient data augmentation is crucial to prevent overfitting and improve generalization capabilities of the system. In the following we outline the data augmentation methods that we combined during model training. All augmentation methods are performed on the fly during training yielding an extremely large number of possible training samples.

\subsection{Mixup}

Mixup [12] is a data augmentation technique originating from classification tasks where a new training sample is generated as a
Table 1: Convolutional Recurrent Neural Network for Audio Tagging with output shapes of each block. Each ConvXd uses a kernel size of three and a stride of one and includes BatchNorm and ReLU.

\begin{tabular}{|c|c|}
\hline Block & output shape \\
\hline LogMel(128) & $B \times 1 \times 128 \times N$ \\
\hline GlobalNorm & $B \times 1 \times 128 \times N$ \\
\hline $2 \times$ Conv $2 \mathrm{~d}(16)$ & $\overline{B \times 16 \times 128 \times N}$ \\
\hline Pool $2 \mathrm{~d}(2 \times 2)$ & $B \times 16 \times 64 \times\lceil N / 2\rceil$ \\
\hline $2 \times$ Conv $2 d(32)$ & $B \times 32 \times 64 \times\lceil N / 2\rceil$ \\
\hline Pool $2 \mathrm{~d}(2 \times 2)$ & $B \times 32 \times 32 \times\lceil N / 4\rceil$ \\
\hline $2 \times$ Conv $2 d(64)$ & $B \times 64 \times 32 \times\lceil N / 4\rceil$ \\
\hline Pool2d $(2 \times 2)$ & $B \times 64 \times 16 \times\lceil N / 8\rceil$ \\
\hline $2 \times$ Conv $2 \mathrm{~d}(128)$ & $B \times 128 \times 16 \times\lceil N / 8$ \\
\hline Pool $2 d(2 \times 1)$ & $B \times 128 \times 8 \times\lceil N / 8\rceil$ \\
\hline Conv2d(256) & $B \times 256 \times 8 \times\lceil N / 8\rceil$ \\
\hline Pool $2 \mathrm{~d}(2 \times 1)$ & $B \times 256 \times 4 \times\lceil N / 8\rceil$ \\
\hline Reshape & $B \times 1024 \times\lceil N / 8\rceil$ \\
\hline $3 \times$ Conv1d $(256)$ & $B \times 256 \times\lceil N / 8\rceil$ \\
\hline $2 \times \mathrm{GRU}(256)$ & $B \times 256$ \\
\hline $\mathrm{fc}_{\mathrm{ReLU}}(256)$ & $B \times 256$ \\
\hline $\mathrm{fc}_{\text {Sigmoid }}(80)$ & $B \times 80$ \\
\hline
\end{tabular}

weighted average of two samples from the dataset:

$$
\tilde{\mathbf{x}}_{i}=\lambda \mathbf{x}_{i}+(1-\lambda) \mathbf{x}_{j}, \lambda \in[0,1]
$$

Similarly their one-hot encoded targets are combined to a soft target vector:

$$
\tilde{\mathbf{y}}_{i}=\lambda \mathbf{y}_{i}+(1-\lambda) \mathbf{y}_{j} .
$$

Although for classification tasks mixup results in ambiguous samples (which probably wouldn't make a lot of sense to a human either) it has shown to improve generalization and robustness of the trained classifier networks. Mixup has successfully been used for general purpose audio tagging, e.g., in [9] in the DCASE 2018 Challenge.

For audio tagging (as opposed to classification) the input audio may already be a superposition of multiple sources. Thus, mixing two or more audio signals together yields a new valid audio signal with an increased number of active events. Therefore, instead of building a weighted average we superpose two waveforms as follows:

$$
\tilde{x}_{i}(t)=\lambda_{0} x_{i}(t)+\gamma \lambda_{1} \frac{\max \left(\left|x_{i}\right|\right)}{\max \left(\left|x_{j}\right|\right)} x_{j}(t-\tau)
$$

with

$$
\begin{aligned}
\gamma & \sim \mathcal{B}(2 / 3), \\
\tau & \sim \mathcal{U}\left(\max \left(-T_{j}, T_{i}-30 \mathrm{~s}\right), \min \left(T_{i}, 30 \mathrm{~s}-T_{j}\right)\right), \\
T_{j} & \leq 1.1 \cdot T_{i}, \\
\lambda_{m} & =a_{m}^{2 b_{m}-1} ; a_{m} \sim \mathcal{U}(1,2) ; b_{m} \sim \mathcal{B}(1 / 2) ; m \in\{0,1\}
\end{aligned}
$$

where $\mathcal{B}$ denotes the Bernoulli distribution. Putting this equation into words we

- perform mixup only with a probability of $2 / 3$,

- only mixup signals which are shorter than 1.1 times the base signal $x_{i}$

- allow mixup to lengthen the signal as long as it does not exceed the maximum length of $30 \mathrm{~s}$,

- normalize signals to the maximum value of the base signal $x_{i}$,

- attenuate or amplify each normalized signal by a random factor. 
We also do not build a weighted average of the individual targets, but simply combine all tags into a single $n$-hot encoded target $\tilde{\mathbf{y}}_{i}$.

\subsection{Frequency Warping}

Recently, SpecAugment [13] was introduced as a simple yet efficient augmentation method of log-mel spectrograms for automatic speech recognition. It uses three different distortions namely time warping, frequency masking, and time masking. In our experiments, however, we found that for audio tagging warping the spectrogram on the frequency axis yielded better performance than time warping. Hence, we exchange the time warping by frequency warping in our version of SpecAugment which is explained in this and the following two sections.

We consider the log-mel spectrogram as an image here with width $T$ and height $F$. Warping the vertical (frequency) axis of the image is controlled by the cutoff frequency

$$
f_{\mathrm{c}} \sim \mathcal{E}(0.5 \cdot F),
$$

where $\mathcal{E}$ denotes the exponential distribution parameterized by the scale $\beta=0.5 \cdot F$, and by the warping factor

$$
\alpha=(1+u)^{2 s-1} ; \quad u \sim \mathcal{E}(0.07) ; s \sim \mathcal{B}(1 / 2) .
$$

Fig. 1 shows the resulting piece-wise linear warping function. Note that $f_{c}$ can be larger than $F$, which results in pure stretching/compressing the whole spectrogram.

It is worth noting that the frequency warping performed here is very similar to Vocal Tract Length Pertubation [14].

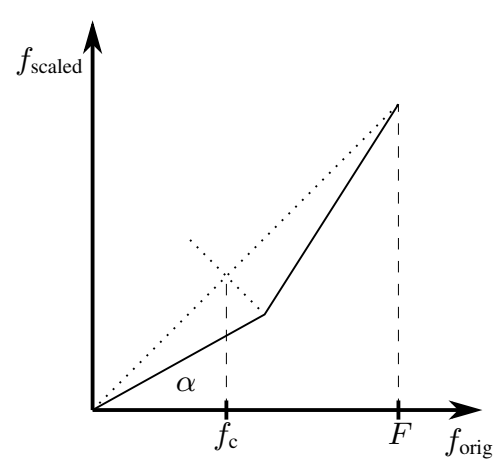

Figure 1: piece-wise linear frequency warping function.

\subsection{Frequency Masking}

We randomly mask $H$ consecutive mel frequencies in the range $\left[f_{0}, f_{0}+H\right]$, where $H$ and $f_{0}$ are drawn from uniform distributions

with $H_{\max }=16$.

$$
\begin{aligned}
H & \sim \mathcal{U}\left(0, H_{\max }\right) \\
f_{0} & \sim \mathcal{U}(0, F-H)
\end{aligned}
$$

\subsection{Time Masking}

We randomly mask $W$ consecutive time frames in the range $\left[n_{0}, n_{0}+W\right]$, where $W$ and $n_{0}$ are drawn from uniform distributions

$$
\begin{aligned}
W & \sim \mathcal{U}\left(0, \min \left(W_{\max }, p \cdot N\right)\right) \\
n_{0} & \sim \mathcal{U}(0, T-W)
\end{aligned}
$$

with $W_{\max }=70$ and $p=0.2$.

\subsection{Data}

\section{TRAINING}

For training we use both the data with curated labels as well as the data with noisy labels.

As the provided train portion with noisy labels primarily contains audio signals of length $15 \mathrm{~s}$ and our model processes whole sequences, there is a bias towards sequences with a length of $15 \mathrm{~s}$. To overcome this bias, we generate, before starting training, new audio excerpts of varying lengths by randomly splitting each of the audio signals with noisy labels into two at length $r_{m} \cdot T_{m}$ with $T_{m}$ being the length of the $m$-th signal and $r_{m} \sim \mathcal{U}(0.1,0.9)$. This results in audio excerpt lengths which are approximately uniformly distributed between $1 \mathrm{~s}$ and $13.5 \mathrm{~s}$. As mixup data augmentation may lengthen audio signals, mixup of the noisy excerpts yields signals distributed between $1 \mathrm{~s}$ and $27 \mathrm{~s}$. Each audio excerpt copies the event tags of the original audio, which results in some additional label noise. The random splitting is performed three times resulting in three different datasets which we refer to as splits $0-2$ in the following ${ }^{3}$.

We mixup curated only data which we refer to as the curated portion in the following as well as we mixup combined curated and noisy data which we refer to as the noisy portion in the following. In each training epoch the curated portion is repeated an integer number of times to prevent training from being dominated by noisy labels. The ratio of noisy labels to the total number of labels in one epoch is referred to as $R=\frac{M_{\text {noisy }}}{M_{\text {total }}}$.

\subsection{Optimization}

The training criterion is the binary cross entropy between the model predictions $\hat{\mathbf{y}}$ and the $n$-hot target vector $\tilde{\mathbf{y}}$ :

$$
L(\hat{\mathbf{y}}, \tilde{\mathbf{y}})=-\sum_{k=0}^{K-1}\left(\tilde{y}_{k} \log \left(\hat{y}_{k}\right)+\left(1-\tilde{y}_{k}\right) \log \left(1-\hat{y}_{k}\right)\right)
$$

with $K=80$ denoting the number of target event classes.

We randomly sample mini batches of size 16 from the training data such that

1. no signal in the mini batch is padded by more than $20 \%$,

2. no example in the mini batch includes the same events as another example in the same minibatch,

and compute gradients of the average loss in the mini batch. We clip gradients at a threshold of 15 . Adam [15] was used for optimization. Training is performed for $200 \mathrm{~K}$ iterations with a learning rate of $3 \cdot 10^{-4}$.

We perform Stochastic Weight Averaging (SWA) [16] for the last $50 \mathrm{~K}$ iterations with a frequency of $1 \mathrm{~K}$ iterations. At the end of training we exchange the model weights for the averaged weights. Finally we update the statistics of all batch normalization layers by making a forward pass on our (unaugmented) training data using the SWA model. SWA has shown to improve generalization and hence performance on unseen data [16]. Another advantage is that with SWA there is no need for held-out data to determine the best performing checkpoint.

\section{EXPERIMENTS}

In the following we evaluate the usefulness of the proposed data augmentation techniques and different methods for label noise handling. While Section 6.1 and Section 6.2 only report the performance of single model systems (which were trained on split 0),

\footnotetext{
${ }^{3}$ The generated splits are available at https://github.com/fgnt/ upb_audio_tagging_2019
} 
Section 6.3 presents performance of ensembles combining models trained on different splits.

System performance is measured in terms of label-weighted label-ranking average precision (lwlrap) evaluated on the private test set. The lwlrap metric measures the capablity of the model to rank active events over non-active events (see [10] for details). The reported scores are one-shot results and were obtained from (late) submissions on the Kaggle competition website.

\subsection{Data Augmentation}

To evaluate the different data augmentation methods we trained single model systems using curated and noisy labeled data using the provided labels. In this section, all models were trained with a noisy label rate of $R=0.56$. Table 2 shows the performance gains due to adding the proposed data augmentation methods. It can be seen that each augmentation method significantly increases the model performance in terms of lwlrap on the private test set.

Table 2: Performance when gradually adding data augmentation methods.

\begin{tabular}{l|c} 
Data Augmentation & lwlrap \\
\hline - & 0.611 \\
Mixup & 0.665 \\
+ Freq. Warp. & 0.701 \\
+ Freq. \& Time Mask. & 0.721
\end{tabular}

\subsection{Label Noise Handling}

In this Section we evaluate different methods for dealing with label noise when using all proposed data augmentation techniques. While for our challenge submission we used relabeling, which is explained first, we adopted Multi Task Learning [17] from the winning team after the challenge has ended, which is explained second.

\subsubsection{Relabeling}

For each of the three splits (as explained in Section 5.1) we trained models on five different folds using a noisy label rate of $R=0.5$. This results in a total of 15 different models which were all combined into an ensemble to make predictions for the noisy excerpts from all three splits. Here the event scores of the individual models were averaged to obtain the ensemble output scores.

For each event we then determined the decision threshold yielding the best error rate jointly evaluated on the set of all noisy excerpts from all splits. These decision thresholds were used to relabel the noisy excerpts, where excerpts without any active event were discarded.

We then used the whole relabeled data of a split for training a new model as we do not need held-out data to determine the best checkpoint due to SWA.

\subsubsection{Multi Task Learning}

The winning team of the DCASE 2019 Challenge Task 2 proposed Multi Task Learning (MTL) to deal with noisy labels [17]. Here different classifier layers are used during training to predict curated and noisy labels, respectively. After the challenge ended we adopted this approach for our model, i.e., we trained different fully connected layers (the last two layers in Table 1) for curated and noisy labels. At test time only the classifier layers trained on the curated labels were used to make predictions.

\subsubsection{Results}

Results for the different methods of label noise handling are shown in Table 3. It can be seen that using the noisy labels results in a significant performance gain. However, using relabeling and MTL yield only small improvement of the lwlrap. In the next section these methods are further evaluated when performing ensembling.

Table 3: Performance for different treatments of label noise.

\begin{tabular}{cc|c} 
Method & $R$ & lwlrap \\
\hline Curated only & 0.00 & 0.687 \\
Provided labels & 0.56 & 0.721 \\
Relabeled & 0.62 & 0.722 \\
MTL & 0.56 & 0.724
\end{tabular}

\subsection{Ensembling}

Finally, we evaluate ensembles of different models. In particular, we combine models trained on different splits and with different noisy label rates. System combination is achieved by averaging the output scores of the contributing systems. Each row in Table 4-6 adds three models which were trained on the three different splits. The results marked by an asterisk in Table 5 represent our submissions to the challenge. It can be seen that neither relabeling nor multi-task learning individually improve performance over the provided labels. Combining all the models from Tables 4-6 into a large ensemble of 27 models, however, raises performance to $76.5 \%$ lwlrap, setting a new state-of-the-art for this task as shown in Table 7. Do note that this ensemble has a total excecution time $<2 \mathrm{~h}$ using CPU, which meets the challenge constraints.

Table 4: Provided labels
\begin{tabular}{cc|c} 
\#models & $R$ & lwlrap \\
\hline 3 & 0.56 & - \\
+3 & 0.64 & - \\
+3 & 0.75 & 0.759
\end{tabular}

Table 5: Relabeled

\begin{tabular}{cc|c} 
\#models & $R$ & lwlrap \\
\hline 3 & 0.62 & $0.746^{*}$ \\
+3 & 0.77 & $0.755^{*}$ \\
+3 & 0.53 & 0.757
\end{tabular}

Table 7: System Comparison

\begin{tabular}{cc|ccc|c}
\multicolumn{3}{c|}{ Table 6: MTL } & & System & lwlrap \\
\cline { 1 - 2 } \#models & $R$ & lwlrap & & Baseline [10] & 0.537 \\
3 & 0.56 & - & & DCASE winner [17] & 0.758 \\
+3 & 0.64 & - & & Kaggle winner & \\
+3 & 0.75 & 0.759 & & Our best subm. [18] & 0.760 \\
& & & & Our best late subm. & $\mathbf{0 . 7 6 5}$
\end{tabular}

\section{CONCLUSIONS}

In this paper we presented our system for the DCASE 2019 Challenge Task 2. Our experiments carried out on the "FSDKaggle2019" data highlight the importance of data augmentation techniques for achieving high classification performance. On the other hand, particular consideration of the label noise did not prove effective in our case. Furthermore, performance was boosted by system combination raising the performance from 0.724 lwlrap of our best single model system to 0.765 by combining a total of 27 models, setting a new state of the art for this task.

\footnotetext{
${ }^{4}$ https: //www.kaggle.com/c/freesound-audio-tagging$2019 /$ leaderboard
} 


\section{REFERENCES}

[1] T. Virtanen, M. D. Plumbley, and D. Ellis, Computational analysis of sound scenes and events. Springer, 2018.

[2] J. F. Gemmeke, D. P. Ellis, D. Freedman, A. Jansen, W. Lawrence, R. C. Moore, M. Plakal, and M. Ritter, "Audio set: An ontology and human-labeled dataset for audio events," in 2017 IEEE International Conference on Acoustics, Speech and Signal Processing (ICASSP). IEEE, 2017, pp. 776-780.

[3] R. Serizel, N. Turpault, H. Eghbal-Zadeh, and A. P. Shah, "Large-scale weakly labeled semi-supervised sound event detection in domestic environments," arXiv preprint arXiv:1807.10501, 2018.

[4] N. Turpault, R. Serizel, A. Parag Shah, and J. Salamon, "Sound event detection in domestic environments with weakly labeled data and soundscape synthesis," June 2019, working paper or preprint. [Online]. Available: https://hal.inria.fr/hal-02160855

[5] E. Fonseca, M. Plakal, D. P. Ellis, F. Font, X. Favory, and $X$. Serra, "Learning sound event classifiers from web audio with noisy labels," in ICASSP 2019-2019 IEEE International Conference on Acoustics, Speech and Signal Processing (ICASSP). IEEE, 2019, pp. 21-25.

[6] C. Kim, A. Misra, K. Chin, T. Hughes, A. Narayanan, T. Sainath, and M. Bacchiani, "Generation of large-scale simulated utterances in virtual rooms to train deep-neural networks for far-field speech recognition in Google Home," in Proc. INTERSPEECH, 2017, pp. 379-383.

[7] J. Salamon and J. P. Bello, "Deep convolutional neural networks and data augmentation for environmental sound classification," IEEE Signal Processing Letters, vol. 24, no. 3, pp. 279-283, 2017.

[8] T. Inoue, P. Vinayavekhin, S. Wang, D. Wood, N. Greco, and R. Tachibana, "Domestic activities classification based on cnn using shuffling and mixing data augmentation," DCASE 2018 Challenge, 2018.
[9] I.-Y. Jeong and H. Lim, "Audio tagging system using densely connected convolutional networks," in Proceedings of the Detection and Classification of Acoustic Scenes and Events 2018 Workshop (DCASE2018), 2018.

[10] E. Fonseca, M. Plakal, F. Font, D. P. Ellis, and X. Serra, "Audio tagging with noisy labels and minimal supervision," arXiv preprint arXiv:1906.02975, 2019.

[11] E. Fonseca, M. Plakal, F. Font, D. P. W. Ellis, X. Favory, J. Pons, and X. Serra, "General-purpose tagging of freesound audio with audioset labels: Task description, dataset, and baseline," CoRR, vol. abs/1807.09902, 2018. [Online]. Available: http://arxiv.org/abs/1807.09902

[12] H. Zhang, M. Cisse, Y. N. Dauphin, and D. Lopez-Paz, "mixup: Beyond empirical risk minimization," arXiv preprint arXiv:1710.09412, 2017.

[13] D. S. Park, W. Chan, Y. Zhang, C.-C. Chiu, B. Zoph, E. D. Cubuk, and Q. V. Le, "Specaugment: A simple data augmentation method for automatic speech recognition," arXiv preprint arXiv:1904.08779, 2019.

[14] N. Jaitly and G. E. Hinton, "Vocal tract length perturbation (vtlp) improves speech recognition," in Proc. ICML Workshop on Deep Learning for Audio, Speech and Language, vol. 117, 2013.

[15] D. P. Kingma and J. Ba, "Adam: A method for stochastic optimization," arXiv preprint arXiv:1412.6980, 2014.

[16] P. Izmailov, D. Podoprikhin, T. Garipov, D. Vetrov, and A. G. Wilson, "Averaging weights leads to wider optima and better generalization," arXiv preprint arXiv:1803.05407, 2018.

[17] O. Akiyama and J. Sato, "Multitask learning and semisupervised learning with noisy data for audio tagging," DCASE2019 Challenge, Tech. Rep., June 2019.

[18] J. Ebbers and R. Haeb-Umbach, "Convolutional recurrent neural network and data augmentation for audio tagging with noisy labels and minimal supervision," DCASE2019 Challenge, Tech. Rep., June 2019. 